\title{
Analise das Emissões Atmosféricas de Fontes Móveis para a cidade Pelotas - RS
}

\author{
Analysis of Mobile Sources Atmospheric Emissions in Pelotas City
}

\author{
Karen Leandra Ávila da Silva ${ }^{1}$, Marcelo Félix Alonso ${ }^{2}$ e Lucijacy Pereira de Oliveira ${ }^{3}$ \\ ${ }^{1}$ Mestranda em Meteorologia, Departamento de Meteorologia, Universidade Federal de Pelotas, Pelotas, Brasil \\ krenleandra.met@hotmail.com \\ 2Doutor em Meteorologia, Departamento de Meteorologia, Universidade Federal de Pelotas, Brasil \\ ${ }^{3}$ Graduanda em Meteorologia, Departamento de Meteorologia, Universidade Federal de Pelotas, Pelotas, \\ Brasil
}

\begin{abstract}
Resumo
Elaborou-se um inventário de emissões veiculares para a cidade de Pelotas, para o ano base de 2012. Foi realizada uma análise das emissões veiculares dos poluentes $\mathrm{CO}$, $\mathrm{NO}$ e $\mathrm{NMHC}$, principais precursores urbanos de $\mathrm{CO}_{2}$ e $\mathrm{O}_{3}$, seguindo a metodologia proposta pela Agência de Proteção Ambiental (EPA). O levantamento da frota veicular para a cidade de Pelotas foi obtido com os dados do Departamento Nacional de Trânsito (DENATRAN-RS), definindo-se em cinco categorias veiculares (veículos leves, veículos comercial leves, caminhões, motocicleta e ônibus), a categorização pelo combustível consumido (gasolina, álcool, bicombustível e diesel) segue distribuição obtida pela Agência Nacional de Fabricante de Veículos Automotores (ANFAVEA) e os fatores de emissão utilizados, foram corrigidos pelo fator de deterioração, conforme metodologia proposta no Inventário Nacional de Emissões Veiculares. Evidencia-se, os automóveis (que totalizam 59\% da frota de Pelotas) foram responsáveis por aproximadamente $63 \%$ da emissão de $\mathrm{CO}$ e $72 \%$ da emissão de NMHC. Conforme esperado, os veículos pesados (caminhões/ônibus) foram responsáveis por $76 \%$ da emissão de NOx no município. Estimou-se que a frota veicular pelotense emite 11.497 toneladas de CO, 3.727 toneladas de NOxe 2.280 toneladas de NMHC.
\end{abstract}

Palavras-chave: emissões veiculares, poluição atmosférica.

\begin{abstract}
This study aimed to establish an vehicular emissions inventory to the Pelotas city, for the 2012 base year. An analysis of vehicular emissions was proposed to the CO, NOx and NMHC atmospheric pollutants, following the methodology proposed by the Environmental Protection Agency (EPA). The vehicle fleet data was obtained from the National Traffic Department (DENATRAN-RS), resumed in five vehicle categories (light vehicles, light commercial vehicles, trucks, motorcycle and bus). The categorization of fuel consumed (gasoline, alcohol, biofuel and diesel) follows distribution obtained from the National Agency of Vehicle Manufacturer (Anfavea) and emission factors have been corrected by the deterioration factor, according to the methodology proposed in the National Inventory of vehicle emissions. As evidenced in inventory results, automobiles (totaling 59\% of Pelotas fleet) accounted for approximately $63 \%$ of the emission of CO and $72 \%$ of NMHC emissions. As expected, the heavy vehicles (trucks / buses) accounted for $76 \%$ of NOx emissions in the city. It was estimated that the fleet vehicle Pelotas emits 11,497 tonnes of CO, 3,727 tons of NOx and 2,280 tons of NMHC.
\end{abstract}

Keywords: vehicular emissions, air pollution 


\section{Introdução}

O crescimento populacional nas grandes e médias cidades do Brasil ocorreu na década de 70 com o crescimento industrial e econômico associado à falta de infraestrutura e planejamento urbano, acarretando em grandes problemas a saúde humana e ao meio ambiente. As emissões de gases traço tóxicos para a troposfera e os produtos de sua oxidação representam um risco direto a saúde. A produção de níveis elevados de ozônio $\left(\mathrm{O}_{3}\right)$ próximo à superfície é de particular preocupação. Altas concentrações de ozônio são relacionadas com doenças em grupos de risco (SALDIVA et al., 1994, 1995).

A poluição do ar vem sendo considerada pela maioria dos países como o principal agente de degradação ambiental do planeta (FEEMA, 2007), por isso que hoje em dia, ter conhecimento da qualidade do ar de uma região é extremamente importante para a gestão ambiental de uma cidade. As interações que envolvem as emissões de poluentes atmosféricas por fontes fixas (indústrias, queima de lixo, fornos, caldeiras etc) ou móveis (veículos automotores etc), as condições meteorológicas e as características geográficas da região em estudo, determinam a concentração de poluentes atmosféricos.

Com respeito às emissões veiculares fontes de importantes precursores do ozônio nas cidades, o Brasil carece de inventários em grande parte das capitais e demais centros urbanos de representatividade econômica. Em outras palavras o país possui poucos estudos quando comparados ao número de médias e grandes cidades existentes. Atualmente podemos citar alguns estados brasileiros que possuem inventário de emissões veiculares, que são: São Paulo, Espírito Santo, Rio de Janeiro e Rio Grande do Sul.

No caso do Rio Grande do Sul, existem alguns inventários de emissões atmosféricas de fontes móveis para a região metropolitana de Porto Alegre (FEPAM, 2010; TEIXEIRA et al., 2008). No entanto, não se tem conhecimento de um inventário veicular para a cidade de Pelotas (região sul do RS) e região, que economicamente é significativa para o estado e está em pleno desenvolvimento. A expansão socioeconômica da região sul do estado, mais especificamente no eixo Rio Grande - Pelotas, associada com o aumento da frota veicular e episódios de poluição, motivou a elaboração de um inventário de emissões veiculares para a cidade de Pelotas, para ano base 2012.

Portanto, pretende-se elaborar um inventário de emissões veiculares para Pelotas, com o intuito de colaborar com o diagnóstico e prognóstico da qualidade do ar no estado do Rio Grande do Sul e contribuir para a geração de um documento diagnóstico básico para tomadas de decisões no município.

\section{Materiais e Métodos}

Para a realização deste inventário utilizou-se a metodologia bottom-up. A análise das emissões dos poluentes hidrocarbonetos não-metano (NMHC), monóxido de carbono (CO), óxidos de nitrogênio $(\mathrm{NO} \times)$ e material particulado (MP) seguiu metodologia proposta pela Cetesb, baseada na Agência de Proteção Ambiental Norte-americana (EPA). Essa metodologia consiste no levantamento das emissões (E) por tipo de combustível (c), por espécie de poluente $(\mathrm{p})$, para um determinado ano de estudo $(\mathrm{t})$, considerando o número de veículos da frota circulante $\left(\mathrm{NV}_{\mathrm{i}, \mathrm{t})}\right.$, a distância média anual percorrida $\left(\mathrm{DM}_{\mathrm{i}, \mathrm{t}}\right)$ por esta frota e os fatores de emissão médios corrigidos de cada poluente $\left(\mathrm{FEC}_{\mathrm{i}, \mathrm{p}, \mathrm{c}}\right.$ ), que são desagregados por ano de fabricação do veículo (i),como mostra a equação 1.

$$
\mathrm{E}_{t}=\Sigma\left(N V_{i, t .} . D M_{i, t} . F e c_{i, p, c}\right)_{\mathcal{c}, \mathrm{p}}
$$

Onde

$\mathrm{E}=$ emissões

$\mathrm{NV}=$ número de veículos

$\mathrm{DM}=$ distância média anual

$\mathrm{Fec}=$ Fator de emissões corrigidas

Os fatores de emissão utilizados foram baseados nos dados para fatores médios de emissões para veículos novos (CETESB, 2009), corrigidos por fatores de deterioração, conforme proposto pela EPA (MCT, 2002). As etapas da metodologia são mostradas na Figura 1. 


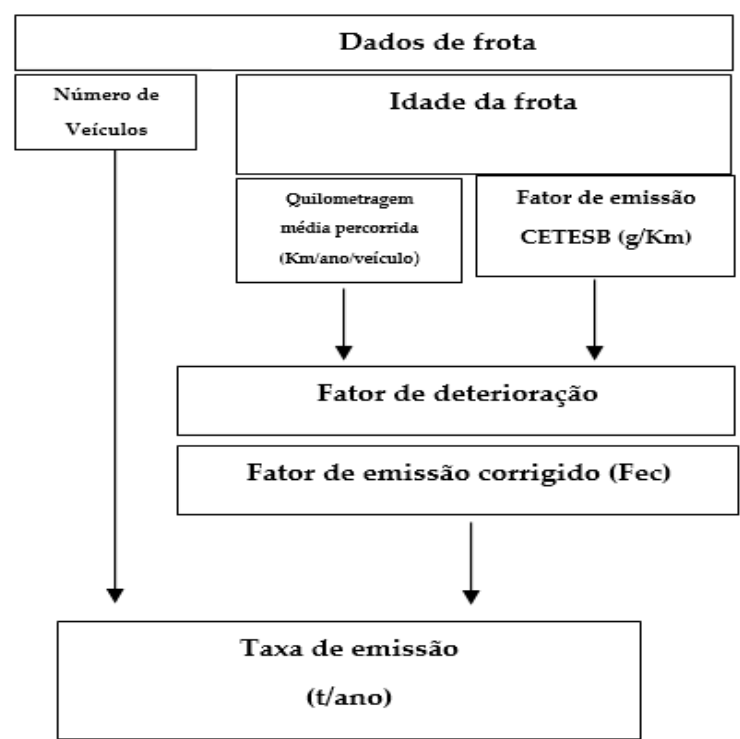

Figura 1: Diagrama esquemático com as etapas da metodologia proposta.

\subsection{Dados da Frota}

Para este trabalho foram definidas cinco categorias veiculares: veículos leves (automóveis); veículos comercias leves (microônibus, camionetes e camionetas), motocicletas, caminhões (caminhões, caminhões trator e trator rodas) e ônibus (ônibus). A categorização seguiu os inventários regionais e nacionais já existentes, com algumas modificações inerentes à particularidade da cidade de Pelotas (Tabela 1).

O levantamento da frota veicular na cidade de Pelotas segue os dados do Departamento Nacional de Trânsito (DENATRAN http://www.denatran.gov.br/). Como a base de dados municipal não possui informação sobre a idade da frota, assumiremos que esse parâmetro segue a distribuição levantada para o estado do Rio Grande do Sul. Quanto à categorização pelo combustível consumido, assumimos a distribuição obtida pela Agência Nacional de Fabricante de Veículos Automotores (ANFAVEA- http://www.anfavea.com.br/) para a frota nacional.
Tabela 1: Categorização da frota em Pelotas.

\begin{tabular}{|c|c|c|}
\hline Categoria & Motor/Combustivel & Definiçãa \\
\hline Veículos Leves & $\begin{array}{c}\text { Otto/Gasolina C Otto/Etanol } \\
\text { Hidratado Otto/Flex Fuel }\end{array}$ & $\begin{array}{c}\text { Veículo automotor destinado ao } \\
\text { transporte de passageiros, com } \\
\text { capacidade para até oito pessoas, } \\
\text { inclusive o condutor }\end{array}$ \\
\hline $\begin{array}{l}\text { Veículos } \\
\text { comerciais } \\
\text { leves }\end{array}$ & $\begin{array}{c}\text { Otto/Gasolina C Otto/Etanol } \\
\text { Hidratado Otto/Flex Fuel } \\
\text { Diesel }\end{array}$ & $\begin{array}{l}\text { Veículo automotor destinado ao } \\
\text { transporte de pessoas ou carga, } \\
\text { com peso bruto de até } 3.500 \mathrm{~kg}\end{array}$ \\
\hline Motocicletas & $\begin{array}{c}\text { Otto/Gasolina C } \\
\text { Otto/FlexFuel }\end{array}$ & $\begin{array}{l}\text { Veículo automotor de duas rodas, } \\
\text { com ou sem side-car, dirigido em } \\
\text { posição montada }\end{array}$ \\
\hline Caminhões & Diesel & $\begin{array}{c}\text { Veículo automotor destinado ao } \\
\text { transporte de carga, com } \\
\text { carroçaria, e PBT superior a } \\
3.500 \mathrm{~kg}\end{array}$ \\
\hline Ônibus & Diesel & $\begin{array}{l}\text { Veículo automotor de transporte } \\
\text { coletivo }\end{array}$ \\
\hline
\end{tabular}

*PTB - Peso Bruto Total.

\subsubsection{Automóveis}

Foram considerados veículos licenciados movidos a gasolina, álcool e flex (álcool e gasolina). Os fatores de emissão foram corrigidos para cada poluente utilizando a equação 2 .

$$
\mathrm{Fec}=\mathrm{FD} . \mathrm{FE}
$$

Onde

$\mathrm{FEC}=$ fator de emissão corrigido

$\mathrm{FD}=$ Fator de deterioração

$\mathrm{FE}=$ Fator de Emissão

Para obter FD, adotou-se a metodologia proposta pela EPA, utilizada em muitos inventários brasileiros (LIMA et al., 2009; UEDA 2008). O equacionamento utilizado para o cálculo do FD em automóveis com ano de fabricação após 1977 é apresentado na Tabela 2.

Tabela 2: Metodologia de cálculo do FD proposta

\begin{tabular}{c|c|c|c}
\multicolumn{5}{|c}{ pela EPA. } \\
\hline Ano de Fabricação & HC & CO & NOx \\
\hline Após 1977 & FDHC $=\frac{4,43+0,25 y}{4,43}$ & FDCO $=\frac{56,34+2,55 y}{56,34}$ & 1 \\
& & & \\
\hline
\end{tabular}


Sendo FDco o fator de deterioração para monóxido de carbono e FDnmн fator de deterioração para hidrocarboneto. O parâmetro Y se obtém através da equação 3.

$$
\mathbf{Y}=\left\{\begin{array}{cc}
\frac{\text { KMacum }}{1,61(10.000)}, & \text { se } K m<100.000 \\
6,21, & \text { se } K m>100.000
\end{array}\right.
$$

E representa a razão entre a quilometragem acumulada e dez mil milhas. A EPA assume que o valor de Y é constante após $100.000 \mathrm{~km}$ (MCT, 2002).

A quilometragem média foi obtida através de amostragem ou de desagregação do inventário estadual (FEPAM, 2010). Em última instância, foram adotados os valores de referência da distância média anual percorrida estimados pela Cetesb em seus inventários. Essa metodologia foi estudada para cada categoria.

Os fatores de emissão para NMHC foram baseados em dois processos distintos: escapamento (NMHCescap), que contempla a emissão devido a combustão incompleta e o processo evaporativo (NMHCevap) que contempla a emissão oriunda da evaporação do cárter.

\subsubsection{Veículos comerciais leves}

Foram considerados veículos comerciais leves: micro-ônibus, camionetes e camionetas, categorizados por tipo de combustível (gasolina, álcool, diesel e flex.). Os fatores de emissão utilizados nessa categoria, ciclo Otto, foram os mesmos dos veículos leves e para os veículos movidos a Diesel foram considerados os fatores de emissão iguais aos veículos pesados (CETESB, 2009).

O cálculo dos fatores de deterioração para NMHC e CO segue a metodologia proposta para os automóveis.

\subsubsection{Caminhões e ônibus}

Considerou-se que todos os veículos desta categoria são movidos a diesel. Os fatores de emissão foram obtidos nos relatórios da qualidade do ar para o período de 2001 até 2011 (CETESB, 2009), obedecendo inclusive às denominações do Programa de Controle Veicular (PROCONVE) vigentes da Tabela 3.

O cálculo dos fatores de deterioração para NMHC e CO seguiu a mesma metodologia proposta para os automóveis.
Tabela 3: Fatores de emissão de motores para veículos pesados do ciclo diesel

\begin{tabular}{|c|c|c|c|c|}
\hline $\begin{array}{c}\text { Fase } \\
\text { PROCONVE }\end{array}$ & $\begin{array}{c}\mathrm{CO} \\
(\mathrm{g} / \mathrm{k} \mathrm{kh})\end{array}$ & $\begin{array}{l}\mathrm{NMHC} \\
(\mathrm{g} / \mathrm{k} \mathrm{Wh})\end{array}$ & $\begin{array}{c}\mathrm{NOx} \\
(\mathrm{g} k \mathrm{kWh})\end{array}$ & $\begin{array}{c}\mathrm{MP} \\
(\mathrm{g} / \mathrm{k} W \mathrm{~h})\end{array}$ \\
\hline $\mathrm{P1}$ & (2) & (2) & (2) & (2) \\
\hline P2 & 1,86 & 0,68 & 10,70 & 0,660 \\
\hline $\mathrm{PB}$ & 1,02 & 0,54 & 6,55 & 0,318 \\
\hline P4 & 0,85 & 0,29 & 6,16 & 0,120 \\
\hline $\mathrm{Pj}$ & 0,86 & 0,16 & 4,67 & 0,078 \\
\hline $\mathrm{P} 6$ & nd & nd & nd & nd \\
\hline
\end{tabular}

2 - Na Fase P1, nenhum destes poluentes era controlado, apenas a emissão de fumaça em regime de carga. nd - não disponível.

\section{Fonte: CETESB (2009)}

\subsubsection{Motocicletas}

Esta categoria engloba as motocicletas e as motonetas. Para estimar os fatores de emissões consideramos que as motocicletas são menores ou iguais a 150 cilindradas e todas de fabricação nacional. O cálculo dos fatores de deterioração para NMHC e CO seguiu a mesma metodologia proposta para os automóveis.

\section{Resultados}

Para a elaboração deste inventário, foram calculadas as emissões para o monóxido de carbono (CO), Óxidos de Nitrogênio (NOx) e Hidrocarbonetos não - metano (NMHC), por se tratarem de poluentes significativos em área urbana.

\subsection{Frota Veicular}

A distribuição da frota na cidade de Pelotas é de 161.562 veículos, de acordo com a definição das categorias. A frota veicular é dividida em, aproximadamente, $59 \%$ automóveis, 8,3\% comerciais leves, $4,5 \%$ caminhões, $28 \%$ motocicletas e $1 \%$ ônibus conforme mostra a Tabela 4. 
Tabela 4: Distribuição da frota por categoria

\begin{tabular}{c|c|c|c|c|c|c}
\hline Ano & Leves & C. Leves & Camininöes & Motocidetas & Onilbus & Total \\
\hline 2012 & 95,372 & 13,346 & 7,322 & 44,433 & 1.089 & 16.1562 \\
\hline$\%$ & 59,031 & 8,661 & 4,339 & 27,502 & 0,674 & 100 \\
\hline
\end{tabular}

Fonte: DENATRAN-RS (2012)

Definiu-se a distribuição dos veículos por combustível utilizando-se os dados nacionais da ANFAVEA. Os resultados da distribuição do total de veículos por categoria e por combustível são apresentados na Tabela 5. Do total de veículos da frota, $72 \%$ é a gasolina, $2 \%$ a álcool, $8 \%$ a diesel

Tabela 5: Distribuição da frota por categoria e combustível.

\begin{tabular}{c|c|c|c|c|c}
\hline & Gasolina & Álcool & Diesel & Total Flex & Total \\
\hline Motocicletas & $44.433(100 \%)$ & $\cdot$ & $\cdot$ & $\cdot$ & 44.433 \\
\hline Automóveis & $65.321(68 \%)$ & $2.922(3 \%)$ & $1.076(1 \%)$ & $26.053(27 \%)$ & 95372 \\
\hline C. Leves & $65.321(47 \%)$ & $2.922(3 \%)$ & $3.300(25 \%)$ & $33.79(25 \%)$ & 13.346 \\
\hline Caminhões & $\cdot$ & $\cdot$ & $9.971(100 \%)$ & $\cdot$ & 9.971 \\
\hline Ônibus & $\cdot$ & $\cdot$ & $1.089(100 \%)$ & & 1.089 \\
\hline
\end{tabular}

Com relação à distribuição dos veículos por idade (Figura 2), nota-se que a frota veicular gaúcha é relativamente antiga, isto é, cerca de $50 \%$ dos veículos fabricados antes de 2001(veículos com mais de 10 anos) ainda estão em circulação. A tendência da frota por idade em Pelotas segue o padrão estadual apresentando ligeira diferença no perfil mais novo (veículos fabricados entre 2009 e 2012).

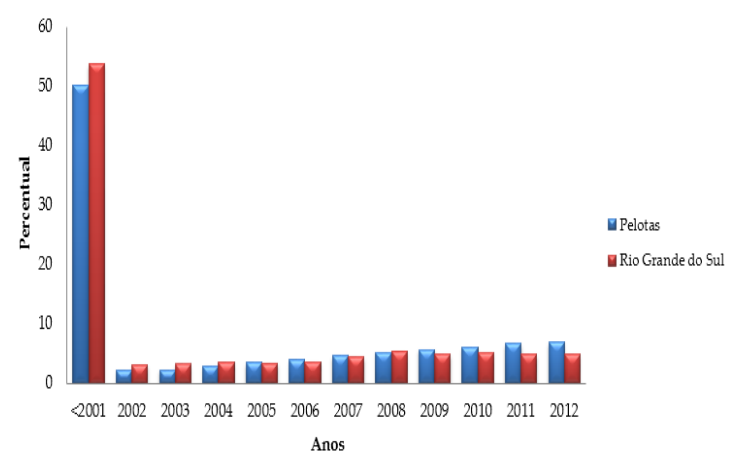

Figura 2: Percentual da distribuição dos veículos por idade no período de <2001 até 2012 Pelotas (Azul) e Rio Grande do Sul (vermelho).

Fonte: DENATRAN (2012).
As emissões para $\mathrm{CO}, \mathrm{NO}_{x}$ e NMHC foram estimadas de acordo com a metodologia proposta pela CETESB, baseada na EPA. Para cada categoria utilizou-se os fatores de emissões e intensidade de uso que melhor se ajustassem a realidade de frota veicular em Pelotas. $\mathrm{O}$ ano base é 2012 e os cálculos das emissões estão representados em toneladas/ ano.

Os resultados mostraram que a frota veicular emite aproximadamente 11.947 toneladas de CO e 3.727 toneladas de $\mathrm{NO}_{\text {x. Com relação aos }}$ hidrocarbonetos não - metanos, estimou-se 1.447 toneladas por emissão dos escapamentos e 833 toneladas de emissão evaporativa, totalizando 2.280 toneladas (Tabela 6).

Observando a Figura 3, podemos notar que os automóveis (que totalizam $59 \%$ da frota) foram responsáveis por aproximadamente $63 \%$ da emissão de CO no município, as motocicletas (que representam $27 \%$ da frota) também apresentaram emissões consideráveis $(28 \%$ da emissão total de (O) por possuir altos fatores de emissão para essa espécie.

Para o poluente $\mathrm{NO}_{\mathrm{x}}$, os resultados apontaram que os caminhões emitem $57 \%$ e os ônibus $19 \%$, totalizando em $76 \%$ da emissão de NOx na cidade de Pelotas por veículos pesados Fig.6. Já para o poluente NMHC, os veículos leves foram responsáveis por $72 \%$ da emissão total, sendo $35 \%$ por combustão incompleta do escapamento (NMHCescap) e 37\% por emissão evaporativa (NMHCevap). Salienta-se que as motocicletas foram responsáveis por $18 \%$ da emissão total dessa espécie (Figura 4).

Tabela 6: Total de emissões de CO, NOx e NMHC para cidade de

Pelotas.

\begin{tabular}{|c|c|c|c|c|c|c|}
\hline Emissoès & Automiovels & $\begin{array}{c}\text { Comercialis } \\
\text { Leres }\end{array}$ & Caminhào & Motocideta & Ônibus & Total \\
\hline $\mathrm{CO}$ & 7.517 & 619 & 340 & 3.350 & 112 & 11.947 \\
\hline $\mathrm{NO}$ & 608 & 170 & 2.129 & IA & 726 & 3.727 \\
\hline NYHCescap. & 798 & 107 & 88 & 421 & . & 1.447 \\
\hline NMHCerap. & 771 & 62 & . & - & . & 883 \\
\hline
\end{tabular}




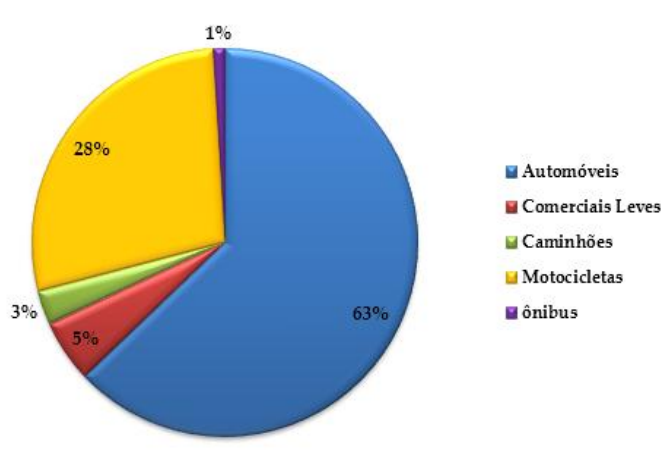

Figura 3: Percentual de emissão de CO para a cidade de Pelota RS.

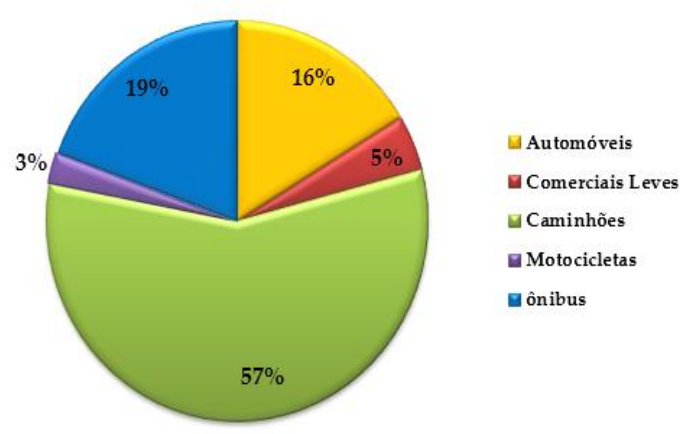

Figura 4: Percentual de emissão de NOx para a cidade de Pelota RS.

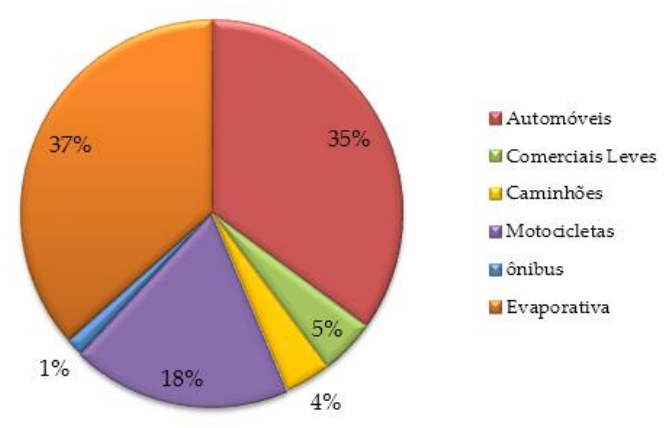

Figura 5: Percentual de emissão de NMHC para a cidade de Pelota RS.

\section{Conclusões}

A partir do levantamento dos dados, foi possível coletar as informações necessárias para a realização do inventário de emissões veiculares para a cidade de Pelotas. Os resultados obtidos neste inventário permitem ter uma visão geral das emissões atmosféricas causadas por fontes móveis na cidade. Estimou-se que o total das emissões veiculares para cada poluente foi de 11.947 toneladas para $\mathrm{CO}, 3.727$ toneladas para $\mathrm{NO}_{x}$ e 2.280 toneladas para NMHC.
Os veículos da categoria leve movidos a gasolina são os maiores contribuintes para a emissão de $\mathrm{CO}$ no município. Este poluente quando lançado na atmosfera pode atuar com o radical hidroxila $(\mathrm{OH})$ para a formação do dióxido de carbono $\left(\mathrm{CO}_{2}\right)$ e em altas concentrações próximas a superfície afeta a saúde humana.

Outro destaque é o impacto dos veículos pesados movidos a diesel, mais especificamente o caminhão, que é o maior emissor de $\mathrm{NO}_{x}$ na cidade. Este poluente é precursor do ozônio em áreas urbanas, juntamente com os hidrocarbonetos não-metano e seu excesso próximo a superfície causa danos diretos a saúde humana.

No entanto, por falta de dados locais mais precisos, alguns parâmetros como intensidade de uso e consumo de combustível foram desagregados de informações estaduais e nacionais, sem incluir a devida especificidade do município. Essa aproximação é válida, no entanto sugere-se o investimento no levantamento, correção e adequação desses parâmetros aos padrões locais de consumo. Também sugere-se estender o estudo para material particulado.

É importante salientar que o uso dos dados de frota veicular licenciada no município não é a aproximação ideal para se capturar os veículos circulantes no ano base. No entanto os órgãos responsáveis carecem de informações de contagem de veículos.

Esse trabalho mostra o quão importante é controlar a qualidade do ar na cidade de Pelotas, através dos programas de inspeção e manutenção de veículos antigos, além de se investir no melhoramento de veículos novos e transportes públicos, pois o aumento da frota veicular na cidade traz preocupações na mobilidade urbana e na área da saúde, já que a poluição gerada por essas fontes está diretamente ligada a doenças.

\section{Referências}

CETESB - Companhia de Tecnologia de Saneamento Ambiental. Relatório de Qualidade do Ar no Estado de São Paulo 2009. São Paulo, 2009 292p 
ENVIRONMENTAL PROTECTION AGENCY (EPA).Compilation of Air Pollutant Emission Factors. 5 ed. Washington, 1999.AP-42, v. 1

FEPAM - Fundação Estadual de Proteção Ambiental Henrique Luiz Roessler - $\mathbf{1}^{\mathbf{0}}$ Inventário de emissões atmosféricas das fontes moveis do Estado do Rio Grande do Sul - Ano Base - 2010.

FEEMA - FUNDAÇÃO ESTADUAL DE ENGENHARIA DO MEIO AMBIENTE. Relatório Anual da Qualidade do Ar. Rio de Janeiro, $2007.91 \mathrm{p}$.

LIMA, Ed. Estimação das Emissões originadas de Veículos leves na cidade de Maringá para o ano de 2005 Acta Scientiarum. Tecnology, Maringá v. 31, n. 1, p. 43-50, 2009

MCT - Ministerio da Ciência e Tecnologia. Emissões de gases de efeito estufa no setor energético por fontes móveis. 2002. Disponivel em

$<$ http://www.mct.gov.br/clima/comunic_old/pdf/ fontesm_p.pdf >. Acesso em: 11 jan. 2013.

SALDIVA, P.H.N.; LICHTENFELS, A.J.F.C.; PAIVA, P.S.O.; BARONE, I.A.;MARTINS, M.A.; MASSAD, E; PEREIRA, J.C.R.; XAVIER, V.P.; SINGER, J.M.; BOHM, G.M. Association between air pollution and mortality due to respiratory diseases in children in São Paulo, Brazil: a preliminary report. Environment Research, v. 65, po 218-225, 1994.

SALDIVA, P.H.N.; POPE, C.A.; SCHWARTZ, J.; DOCKERY, D.W.;LICHTENFELS, A.J.; SALGE, J.M.; BARONE, I.; BOHM, G.M. Air pollution and mortality in elderly People: a time-series study in São Paulo, Brazil. Archives of Environmental Health, v. 50, n. 2, 159-163, 1995.

TEIXEIRA, E. Estudo das emissões de fontes móveis na região metropolitana de porto alegre, rio grande do sul Química. Nova, v. 31, n. 2, p. 244-248, 2008

UEDA, C. Inventário de Emissões de Fontes Veiculares da Região Metropolitana de Campinas, São Paulo Química. Nova, v. 34, n. 9, p. 1496-248, 2008. 